АНАЛИЗ ФАКТОРОВ, ПРЕПЯТСТВУЮЩИХ ФОРМИРОВАНИЮ ПРИВЕРЖЕННОСТИ ЛЕЧЕНИЮ СРЕДИ БОЛЬНЫХ САХАРНЫМ ДИАБЕТОМ, И СТРАТЕГИЙ, СПОСОБСТВУЮЩИХ ЕЕ ПОВЫШЕНИЮ

( С Н.В. Лиходей ${ }^{1}$, М.Ф. Калашникова' ${ }^{1}$ Е.М. Лиходей², В.В. Фадеев ${ }^{1}$

'ФГАОУ ВО ПервыЙ Московский государственный медицинский университет им. И.М. Сеченова (Сеченовский Университет) Минздрава России, Москва

ФГБОУ ВО Российский национальный исследовательский медицинский университет им. Н.И. Пирогова Минздрава России, Москва

В обзоре рассмотрен современный взгляд на проблему низкой приверженности лечению среди пациентов, страдающих хроническими заболеваниями, в частности сахарным диабетом 2 типа (СД2). Согласно определению Всемирной организации здравоохранения, «приверженность лечению» - это степень соответствия поведения пациента назначениям врача в отношении приема лекарственных препаратов, выполнения рекомендаций по питанию и/или изменения образа жизни. Современная медицинская литература насчитывает большое число научных публикаций, посвященных изучению различных факторов, обуславливающих низкую приверженность лечению. Для их обозначения наиболее часто используется термин «барьеры». В первой части работы проведен анализ основных факторов, препятствующих соблюдению рекомендаций врача, включающих социально-экономические, психологические (личностные), барьеры, связанные с самим заболеванием, особенностями его лечения, с организацией медицинской помощи (системой здравоохранения).

Во второй части обзора рассматриваются различные теоретические модели поведения пациентов и стратегии, способствующие улучшению приверженности лечению. По мнению большинства исследователей, при СД2 наблюдается неудовлетворительная (низкая) приверженность лечению, и ни одна из существующих интервенционных стратегий не может улучшить приверженность лечению среди всех пациентов. Краеугольным камнем всей системы управления СД является обучение больных в рамках разработанных структурированных программ. С другой стороны, успех зависит от индивидуального подхода, течения болезни и обязательного учета индивидуальных психологических особенностей каждого человека. Установление партнерских доверительных взаимоотношений между врачом и пациентом способствует формированию большей удовлетворенности пациентов лечением, улучшению приверженности и, в конечном счете, оказывает влияние на эффективность лечения и клинические исходы.

КЛЮЧЕВЫЕ СЛОВА: сахарный диабет 2 типа; барьеры; приверженность лечению; теоретические модели поведения

\title{
ANALYSIS OF THE FACTORS THAT PREVENT ADHERENCE TO TREATMENT IN PATIENTS WITH DIABETES MELLITUS AND THE STRATEGIES THAT CONTRIBUTE TO THE IMPROVEMENT IN ADHERENCE
}

(c) Natalia V. Likhodey' ${ }^{1}$ Marina F. Kalashnikova', Elena M. Likhodey² ${ }^{2}$ Valentin V. Fadeyev ${ }^{1}$

1.M. Sechenov First Moscow State Medical University, Moscow, Russia

${ }^{2}$ Pirogov Russian National Research Medical University, Moscow, Russia

This review examined the current problem of low adherence to treatment in patients with chronic diseases, particularly type 2 diabetes mellitus. According to the definition of the World Health Organization, 'adherence to treatment' is the degree to which a patient's behaviour corresponds to the doctor's recommendations with respect to medications and implementation of dietary advice and/or lifestyle changes. The current medical literature includes a large number of scientific publications devoted to the study of various factors that lead to low adherence to treatment. The term 'barriers' is most often used to designate these factors. The first part of this work contains an analysis of the main factors that impede compliance to the doctor's recommendations, such as socioeconomic and psychological (personal) barriers related to the disease itself, the peculiarities of its treatment and the organisation of medical care (the health care system).

The second part of this review examines the different theoretical models of patient behaviour and strategies that improve adherence to treatment. Most researchers believe that there is an unsatisfactory (low) adherence to treatment and that none of the existing intervention strategies can improve adherence to treatment among all patients. The cornerstone of the entire diabetes management system is the training of patients within the framework of developed structured programmes. Conversely ${ }_{\prime \prime}$ success depends on the individual approach, the course of the disease and the mandatory consideration of the individual psychological characteristics of each person. Establishment of a partnership built on trust between a doctor and a patient contributes to greater patient satisfaction with treatment and improved adherence, and this relationship ultimately affects the treatment efficacy and clinical outcomes.

KEYWORDS: type 2 diabetes mellitus; barriers; patient compliance; theoretical models 
Сахарный диабет 2 типа (СД2) является одним из самых распространенных хронических заболеваний, приводящим к развитию микро- и макрососудистых осложнений и оказывающим негативное влияние на все сферы жизни больных. В основе современной тактики лечения данного заболевания лежит многофакторный подход, включающий структурированное обучение пациентов принципам здорового образа жизни и навыкам проведения самоконтроля гликемии, пожизненный прием сахароснижающих препаратов (ССП), проведение мероприятий по профилактике развития и замедлению прогрессирования осложнений СД2, а также лечение сопутствующих заболеваний.

В последние годы был достигнут существенный прогресс в лечении данного заболевания: в России и в мире разработаны и внедрены в практику алгоритмы оказания специализированной медицинской помощи больным СД, созданы инновационные классы ССП, определены индивидуальные целевые уровни гликемии. Однако до сих пор остается целый ряд нерешенных вопросов в организации специализированной помощи при СД2, среди которых неспособность больных длительно соблюдать рекомендации по лечению и образу жизни (низкая приверженность), что является глобальной медицинской проблемой современности.

Многочисленные научные исследования, посвященные изучению данной проблематики, привели к формированию целостной концепции и специфической терминологии. В середине XX в. Haynes и Sackett ввели понятие «compliance», определяя его как характер поведения пациента по отношению к назначенному медикаментозному лечению [1]. В русскоязычной литературе ему соответствовал термин «комплаентность», предложенный авторами в 1995 г., который трактовался как отношение пациента к лечению и обусловленное этим отношением поведение [2].

В 2001 г. на конференции Всемирной организации здравоохранения (ВО3) экспертным советом был предложен термин «приверженность лечению» (от англ. adherence to treatment - соблюдение, следование), который трактовался как «степень соблюдения пациентом медицинских рекомендаций» [3]. Однако и это определение подверглось критике, так как слово «медицинские» не подходило для описания комплекса мероприятий, применяемых в лечении хронических заболеваний, а термин «рекомендации» (от англ. instructions - рекомендации, назначения, инструкции) означал пассивное выполнение человеком медицинских предписаний в отличие от модели поведения, заключающейся в активном взаимодействии врача и пациента.

В результате в 2003 г. экспертами ВОЗ было принято расширенное определение понятия приверженности лечению больных, страдающих хроническими заболеваниями, которое обозначает степень соответствия поведения пациента (прием лекарственных препаратов, выполнение рекомендаций по питанию и/или изменению образа жизни) врачебным рекомендациям. В резолюции подчеркивается, что высокая приверженность лечению может быть достигнута лишь при тесном сотрудничестве пациента с медицинским работником (врачом, медсестрой или другим медицинским работником) [4]. При этом необходимо создание доверительной атмосферы для обсуждения альтернативных подходов к лечению, проблем, с которыми может столкнуться пациент, и планирования дальнейшего динамического наблюдения.

Более подробное описание принятой в настоящее время терминологии, методов расчета показателя приверженности лечению, общих и специализированных диагностических шкал (опросников), применяемых для оценки приверженности лечению больных с СД2, было опубликовано авторами в журнале «Лечащий врач» в 2015 г. [5]. Настоящая статья посвящена анализу существующих причин («барьеров»), препятствующих соблюдению пациентом врачебных рекомендаций, а также в ней рассматриваются различные психометрические модели, предложенные специалистами для преодоления низкой приверженности лечению.

\section{АНАЛИЗ ФАКТОРОВ (БАРЬЕРОВ), ПРЕПЯТСТВУЮЩИХ ВЫПОЛНЕНИЮ ВРАЧЕБНЫХ РЕКОМЕНДАЦИЙ}

Существует мнение, что несоблюдение пациентами врачебных рекомендаций при СД2 является следствием недостаточно эффективной системы обучения и неэффективного взаимодействия врача и пациента, наряду с низкой мотивацией больных в отношении изменения образа жизни и пожизненного приема большого числа медикаментов. Другими причинами низкой приверженности лечению являются личностные и психологические особенности пациентов, клинические особенности заболевания, характер проводимого лечения, социальноэкономические факторы и особенности организации медицинской помощи в различных странах $[5,6]$.

Современная медицинская литература насчитывает большое число научных публикаций, посвященных изучению причин низкой приверженности лечению. Наиболее часто используется термин «барьеры», под которым понимают ряд факторов различного свойства и рода, влияющих отрицательным образом на способность пациента в надлежащем порядке придерживаться врачебных рекомендаций. Данный термин впервые был введен социальными психологами Hochbaum, Rosenstock, Kegels в 1950-х гг. при описании поведенческой модели Health Belief Model (HBM) [7].

Существенный рост интереса специалистов к проблеме низкой приверженности лечению отмечается в течение последних 15 лет. Учитывая многообразие существующих барьеров, препятствующих соблюдению врачебных рекомендаций, и отсутствие единой общепринятой классификации, в соответствии с мнением пяти ведущих специалистов в табл. 1 представлены основные причины низкой приверженности среди пациентов, страдающих хроническими заболеваниями.

В 2009 г. Gellad и соавт. в своей работе разделили причины неприверженности лечению на основные, дополнительные и медицинские, уделив отдельное внимание проблеме удовлетворенности пациента проводимым лечением [8].

Taha и соавт. более подробно изучали финансовые, физические и психологические барьеры, препятствующие соблюдению диеты и физической нагрузки, выполнению рекомендаций по медикаментозному лечению и проведению диагностических исследований [9]. 
Таблица 1. Барьеры, препятствующие выполнению врачебных рекомендаций

\section{Авторы \\ публикации}

1. Основные: необходимость оплаты пациентом части затрат на лечение, не покрытых полисом медицинского страхования (финансовые барьеры); сложность соблюдения режима лечения; неуверенность пациента в эффективности назначенного курса лечения; наличие депрессии у пациента.

Gellad и соавт.

(2009 г.) [8]

2. Дополнительные: отсутствие достаточных знаний о болезни, способах ее лечения и возможных побочных эффектах проводимой терапии.

3. Медицинские: степень доверия специалисту и удовлетворенность проводимым лечением

Taha и соавт.

(2011 г.) [9]

Финансовые, физические и психологические причины, препятствующие соблюдению диеты и физической нагрузки, выполнению рекомендаций по медикаментозному лечению и проведению диагностических исследований, а также проблема недостатка знаний пациента о своем заболевании.

Наличие психологических проблем, таких как депрессия; наличие когнитивных нарушений; лечение бессимптомной болезни; плохое планирование терапии; побочные эффекты лекарств; от-

White

(2010 г.) [10] сутствие веры пациента в пользу лечения; отсутствие достаточного понимания у пациента его болезни; плохие взаимоотношения между пациентом и его лечащим врачом; наличие барьеров при уходе за пациентом; пропуск запланированных консультаций врача; сложность режима лечения; стоимость лечения (включая высокую доплату за лечение из собственных средств пациента).

1. Барьеры, связанные с личностью пациента (личностные барьеры): демографические факторы (возраст, национальность, пол, образование, семейное положение); психосоциальные факторы (убеждения, мироощущение, наличие мотивации); отношения между пациентом и врачом; компетентность в сфере медицины; физические проблемы пациента; вредные привычки (курение или употребление алкоголя); нарушения памяти (забывчивость); анамнез приверженности лечению.

2. Барьеры, связанные с лечением: способ проведения лечения; сложность режима лечения; продолжительность лечения; побочные эффекты медикаментозного лечения; степень необхо-

Jin и соавт. (2008 г.) [11] димого изменения поведения пациента, связанного с заболеванием; вкус лекарства; требования по хранению лекарств.

3. Социальные и экономические барьеры: невозможность пациентом взять отгул или выходной на работе; соотношение доходов и стоимости лечения; наличие социальной поддержки.

4. Барьеры, связанные с системой здравоохранения: недостаточная доступность медикаментов и врачебной помощи; время ожидания консультации врача или обследований; наличие трудностей при получении лекарств по рецепту; неудовлетворенность пациента врачебной консультацией.

5. Барьеры, связанные с заболеванием: проявления заболевания; степень тяжести заболевания.

1. Демографические: принадлежность к этническому меньшинству, низкий социально-экономический статус и низкий уровень образования.

2. Психологические: понятность смысла проводимой терапии; эффективность, по мнению больного, рекомендаций; уверенность в том, что результаты лечения оправдают затраты на его проведение; убежденность больного в том, что он в состоянии справиться с предписанным режимом лечения, а также психологическая поддержка и помощь окружающих. Важна информированность

Delamater (2006 г.) [12] пациента о состоянии здоровья, понимание серьезности заболевания, уязвимость по отношению к сложностям, отношение к стрессу, раздражительность, депрессия, пищевые расстройства.

3. Социальные: семейные взаимоотношения, напрямую влияющие на приверженность лечению. 4. Медицинские: взаимоотношения с медицинским персоналом, наличие их поддержки, частота контактов, внимательность по отношению к пациенту.

5. Факторы, связанные с заболеванием и его лечением: характер заболевания (хронический или не хронический); очевидность или неочевидность симптомов заболевания; сложность режима и необходимость внесения изменений в образ жизни пациента
White в своей работе провел анализ психологических и социальных причин неприверженности лечению [10].

Большой интерес вызывают работы Jin и соавт. [11] и Delamater [12], которые предложили структурированный подход к описанию существующих барьеров, разделив их на пять основных категорий: социально-демографические; психологические; обусловленные заболеванием и его лечением; экономические; связанные с системой здравоохранения.
Некоторые из перечисленных барьеров являются «немодифицируемыми», такие как демографические и социальные особенности пациента (пол, возраст, национальность, принадлежность к этническому меньшинству, семейное положение, низкий социально-экономический статус, низкий уровень образования), включая факторы, связанные с самим заболеванием и особенностями его лечения (характер заболевания, степень тяжести, очевидность или неочевидность симптомов заболевания) [13]. 
В то же время существует большое число «модифицируемых» факторов, которые можно устранить, что приведет к повышению приверженности лечению. Достижения современной медицины позволяют во многих случаях улучшить приверженность, предложив пациенту более удобный способ лечения; изменить (по возможности) его продолжительность, использовать более эффективные терапевтические стратегии; минимизировать в некоторых случаях побочные эффекты проводимой терапии [12].

Известно, что наличие физических проблем у пациента и нарушение когнитивных функций, особенно среди лиц пожилого и старческого возраста, также могут оказывать существенное влияние на приверженность лечению [11]. В подобных ситуациях крайне важны помощь окружающих и организация специализированного индивидуального медицинского ухода для такой категории больных.

Отдельного рассмотрения заслуживают барьеры, связанные с системой здравоохранения, которые во многом определяются организацией медицинской помощи в различных странах. Наиболее значимыми из них являются финансовые барьеры (необходимость оплаты пациентом части или всех затрат на лечение, не покрытых полисом медицинского страхования), недостаточная доступность медикаментов и врачебной и сестринской помощи; наличие трудностей при получении лекарств по рецепту; время ожидания консультации врача или обследований; неудовлетворенность пациента врачебной консультацией.

Еще одним важным фактором, оказывающим влияние на приверженность лечению и, в конечном счете, на эффективность проводимой терапии, является наличие депрессии. Своевременная диагностика данного состояния и устранение ее проявлений позволяют улучшить прогноз течения основного заболевания [14].

Таким образом, среди всех существующих барьеров, выявление и преодоление модифицируемых факторов могло бы приводить к существенному улучшению приверженности лечению.

Изучение психологических особенностей пациента является предметом пристального внимания специалистов. По мнению Delamater, психологические барьеры включают различные аспекты психосоциального поведения человека: понимание задач проводимой терапии и эффективности рекомендаций; уверенность в том, что результаты лечения оправдают затраты на его проведение; убежденность, что он в состоянии справиться с предписанным режимом лечения [12]. Также большое значение имеют информированность пациента о состоянии своего здоровья и понимание характера и серьезности заболевания.

\section{ТЕОРЕТИЧЕСКИЕ МОДЕЛИ ПОВЕДЕНИЯ ПАЦИЕНТОВ И СТРАТЕГИИ, СПОСОБСТВУЮЩИЕ УЛУЧШЕНИЮ ПРИВЕРЖЕННОСТИ ЛЕЧЕНИЮ}

В конце XX в. специалистами были изучены различные модели поведения больных и разработаны стратегии преодоления низкой приверженности лечению.

В 1980 г. Ajzen и Fishbein представили «Теорию запланированного поведения» (The Theory of Planned
Behaviour), основанную на утверждении, что поведение пациента определяется его отношением к самому лечению (ожидаемая ценность терапии), его субъективным пониманием существующих норм и своих возможностей придерживаться надлежащего поведения [14].

Rosenstock и соавт. изучали основные подходы к изменению поведения населения в медицинских профилактических программах США [15]. Авторы разработали «Модель убеждений о здоровье» (Health Beliefs Model), согласно которой, на поведение больного оказывают влияние четыре фактора:

- какие преимущества он видит в приверженности лечению (например, уменьшившиеся симптомы болезни);

- какие барьеры мешают ему соблюдать рекомендации по лечению (например, побочные эффекты терапии);

- какова его восприимчивость или уязвимость перед болезнью, под которой понимается степень уверенности пациента в возможности предотвращения рецидива заболевания;

- осознанная серьезность болезни (под серьезностью понимают то, насколько сам больной оценивает свое заболевание). В данной работе было сформулировано определение понятия «приверженность лечению», которое является «результатом осмысленного изменения пациентом его поведения и образа жизни, необходимое для выполнения рекомендаций врача».

Prochaska и соавт. предложили свою «Транстеоретическую модель изменения поведения» (The Transtheoretical Model/The Stages Of Change model (SOC)), в которой были выделены пять последовательных стадий изменения поведения больного [16]:

1-я стадия: незаинтересованность (возможность изменения поведения в течение ближайших 6 мес отсутствует);

2-я стадия: заинтересованность (рассмотрение изменения поведения в течение ближайших 6 мес);

3-я стадия: подготовка (планирование изменить поведение в течение следующих 30 дней);

4-я стадия: действие (текущее изменение поведения);

5-я стадия: поддержание (успешное изменение поведения в течение, по меньшей мере, 6 мес).

Определение стадии, на которой находится пациент, позволяет сразу перейти к наиболее эффективным стратегиям изменения поведения с целью повышения приверженности лечению и избежать одновременного использования всех инструментов воздействия (информирование, консультации, напоминания, самоконтроль, семейная терапия, поддержка). Модель SOC также описывает различные эмпирические и поведенческие стратегии, которые ассоциируются с успешным переходом от одной стадии к другой. Эмпирические стратегии отражают когнитивное, оценочное и эмоциональное изменение поведения, в то время как поведенческие стратегии заключаются в использовании напоминаний или поощрений при соблюдении врачебных рекомендаций.

Также авторы выделили основные психологические установки, характеризующие пациента с позиции степени его готовности к изменению поведения. Баланс принятия решения состоит из «за» и «против» изменения в поведении и является идеальным показателем готовности пациента двигаться дальше от исходной стадии незаинтересованности. 
В основе «Модели саморегуляции» (The Self Regulation Model) лежит утверждение, что реакция пациента на болезнь зависит от его собственной оценки этого заболевания, основанной на его знаниях [17]. Центральным звеном этой теории является понимание того, что болезнь (опыт плохого состояния здоровья) отличается от заболевания (состояние, представляющее диагностированную патологию).

Болезнь представляет собой субъективный опыт, который может или не может ассоциироваться с физической патологией (заболеванием). При столкновении с угрозой здоровью пациент должен сформировать для себя личное представление о болезни. Это когнитивное восприятие заболевания идет параллельно с генерированием и обработкой сопутствующих эмоциональных реакций [18].

В дальнейшем при разработке поведенческих моделей особое внимание авторы уделяли изучению стратегий и методов преодоления барьеров неприверженности лечению.

В поведенческой модели SIMPLE авторы разработали подробные инструкции для врача с целью улучшения приверженности лечению в зависимости от тех барьеров, с которыми сталкивается каждый пациент в процессе лечения [19]. Ниже приводим подробное описание рекомендаций для медицинских работников, которые могут помочь при работе с пациентами в клинической практике.

\section{S (Simplifying regimen characteristics) - Упрощение режима}

1. Оптимизируйте время приема лекарственного препарата, кратность приема и его дозировку. Лекарства, принимаемые один раз в день, более предпочтительны, но если стоимость таких лекарств является основным барьером приверженности лечению, то это не решит проблему.

2. Подстройте режим лечения под режим обычной жизни пациента.

3. Рекомендуйте, чтобы все лекарства принимались в одно и то же время дня.

4. Избегайте назначать лекарства, которые требуют соблюдения дополнительных условий приема (например, строгая зависимость от приемов пищи).

5. Разбейте режим на простые шаги, которые могут вводиться последовательно, и убедитесь, что пациент понимает каждый шаг.

6. Используйте вспомогательные средства и сигналы для напоминания о приеме лекарств.

7. Ориентируйтесь на то, чтобы изменить ситуацию, а не пациента (больше пояснений, повторений, облегчений курса лечения). Другими словами, врачу следует приспособить курс лечения под пациента, а не пациента под курс лечения.

\section{I (Imparting knowledge) - Передача знания}

Приверженность повышается, когда пациент понимает свое состояние и преимущества лечения, но передача знания должна комбинироваться с упрощением режима.

1. Стремитесь к совместному принятию решений по выбору терапии.

2. Поощряйте в пациенте ведение дискуссий с врачом, медсестрой и фармацевтом.
3. Обеспечивайте пациента понятными инструкциями (письменными и вербальными) для всех предписаний:

a) ограничьте инструкции до 3-4 основных пунктов;

b) используйте простой, понятный для пациента язык;

с) применяйте письменную информацию или брошюры и т.д.

4. При необходимости вовлекайте в обсуждение семью и друзей пациента.

5. При наличии хронических заболеваний предоставьте пациентам ссылки на качественные интернет-сайты или специальную литературу для обеспечения необходимой дополнительной информацией.

6. Предоставьте конкретный совет в отношении того, как покрыть медицинские затраты.

7. Поддерживайте все обсуждения, особенно с недостаточно информированными о своем заболевании пациентами.

M (Modifying patient beliefs) - Изменение убеждения пациента и его поведения.

1. Уполномочьте пациентов самостоятельно следить за их состоянием:

a) спросите пациента о его проблемах со здоровьем. Создайте открытый диалог с каждым пациентом и спросите о его ожиданиях, нуждах и имеющемся опыте лечения;

b) спросите пациента о том, что может помочь ему стать и оставаться приверженным лечению.

2. Убедитесь, что пациенты понимают, что они могут попасть в зону риска, если они не будут принимать лекарства.

3. Попросите пациентов описать последствия отсутствия приема лекарств.

4. Попросите заново описать преимущества приема лекарств.

5. Обратите внимание на страхи и переживания (приобретенные барьеры) приема лекарств.

6. Рассмотрите использование непредвиденных контрактов. Непредвиденный контракт - это соглашение между пациентом и врачом, которое устанавливает поведенческие цели пациента.

7. Установите вознаграждение за высокую приверженность лечению:

а) вознаградите эффективность лечения при выполнении рекомендаций похвалой;

b) поощряйте маленькими сувенирами, сокращенной частотой визитов.

P (Patient communication) - Установление общения и доверия.

1. Улучшите собственные навыки интервьюирования.

2. Практикуйте активное слушание. Активное слушание является интерактивным процессом с целью услышать смысл и требует большого внимания к словам пациента. Это включает такие техники, как:

а) подтверждение того, что сообщение пациента было услышано (обратная связь, вопросы и другие знаки);

b) правильно интерпретируйте сообщение пациента; 
с) используйте вербальные и невербальные знаки для того, чтобы показать понимание и сочувствие;

d) в ходе обсуждения обеспечивайте пациенту обратную связь;

е) продлите обсуждение для того, чтобы получить больше информации;

f) предоставьте необходимые ответы и посмотрите на его реакцию: согласится ли он или примет с неохотой.

3. Обеспечьте пациента эмоциональной поддержкой.

4. Обеспечьте пациента ясной и точной информацией и заставьте пациента ее повторить.

5. Вовлеките пациента в принятие решений и выявите/ определите размер вклада пациента в принятие решений.

6. Отведите пациенту время на то, чтобы он мог задать вопросы.

7. Постройте доверительные отношения с пациентом. Врачи могут использовать короткий тест для того, чтобы понять, нужно ли им улучшить эту ситуацию.

\section{L (Leaving the bias) - Преодолеть предубеждение.}

Многое доказывает, что этнические и социальноэкономические различия влияют на результат лечения повсеместно. Пациенты в этом случае ощущают меньше внимания и, как следствие, меньше времени для общения с врачом. Для того чтобы преодолеть этнические и социальные барьеры, врачу стоит:

1. оценить уровень медицинских знаний у пациента, его усилия, направленные на улучшение результатов лечения;

2. применять новые знания на практике;

3. оценить уверенность данного пациента в положительном результате лечения;

4. пересмотреть стиль общения для того, чтобы понять, является ли он действительно ориентированным на пациента;

5. признать наличие предубеждений в процессе принятия решений (умышленных или неумышленных);

6. обратить внимание на диссонанс между расой пациента и врача, их этническими различиями и языком:

a) знать демографию расы пациента;

b) взять дополнительное время для преодоления культурных барьеров;

с) отдельно спросить об отношении, убеждениях и культурных нормах касательно курса лечения;

d) использовать культурно и лингвистически подходящие средства вмешательства, ориентированные на пациента, которые могли бы повысить вовлеченность, активность пациента;

е) адаптировать обучение к уровню понимания пациента;

f) использовать наглядные пособия при обучении пациента.

E (Evaluating adherence) - Оиенить приверженность.

1. Самоотчеты являются наиболее распространенным способом оценки приверженности. Спросите пациентов просто и прямо, придерживаются они режима или нет.

2. Спрашивайте о поведении и приверженности при каждой встрече.
3. Выведывайте о барьерах приверженности и недостатке восприимчивости к медицинской информации.

4. Если самоотчет все равно оставляет вопросы, используйте подсчет таблеток или оценку уровня лекарств в сыворотке крови или моче.

5. Периодически просматривайте контейнер лекарств пациента, отмечая изменения.

Важно отметить, что для практического применения данной модели и проведения мотивирующего интервью необходимо обучение врача методологии, используемой в ходе беседы с пациентом, целью которой является осознание пациентом своих проблем, а также его активное участие в их разрешении, и/или привлечение дополнительного специалиста-психолога. Кроме того, применение данной методики в рутинной практике ведения больных с хроническими заболеваниями требует дополнительного времени и связанных с этим материальных расходов.

В заключение данной части обзора следует отметить недавно разработанную «Модель Информирование-Мотивация-Стратегия» (Information-Motivation-Behavioural Skills Model) [20], которая рассматривает влияние когнитивных, психологических и социальных факторов на взаимодействие врача и пациента и предлагает три подхода к достижению взаимопонимания: информирование, мотивацию и разработку стратегии поведения, которые могут быть применены врачом индивидуально для каждого пациента. Информирование представляет собой знания о терапевтических/медицинских аспектах развития болезни, ожидаемом курсе лечения, эффективных стратегиях по ее лечению. Врачу необходимо эффективно доносить информацию до пациента; построить доверительные отношения; поощрять пациента к активному участию в процессе принятия решений; воспринимать его в качестве партнера при назначении лечения; внимательно слушать пациентов, рассказывающих о возникающих проблемах в ходе проводимой терапии. Мотивация включает личное отношение пациента к приверженности, субъективные нормы пациента и его представления о должном поведении. Врачу необходимо помочь пациенту поверить в эффективность лечения; выявлять, слушать и обсуждать любое негативное отношение к лечению. Важно помочь пациенту сформировать ответственное отношение к приверженности и вселить в него уверенность в том, что он способен придерживаться назначенного курса лечения. Стратегия. Поведенческие навыки пациента включают в себя его уверенность в том, что он обладает специфическими знаниями и возможностями, необходимыми для выполнения рекомендаций по лечению, например, возможностью заручиться социальной поддержкой. Врач должен помогать преодолевать выявленные барьеры, стоящие на пути к эффективному выполнению курса лечения; привлекать людей, которые могут предоставить практическую помощь, помогать в поиске ресурсов предоставления финансовой помощи или скидки на лечение (льготу); давать письменные инструкции/напоминания по режиму назначенного курса терапии; подписывать «поведенческое соглашение»; связываться с группами поддержки пациентов с подобными заболеваниями; обеспечивать, по возможности, электронными напоминаниями или телефонными звонками. 
Таким образом, рассмотрев различные модели изменения поведения пациентов, можно заключить, что все предложенные стратегии улучшения приверженности можно разделить на три группы: психолого-педагогические, поведенческие и когнитивно-поведенческие.

Если опираться только на рассмотренные выше модели, то общая схема повышения приверженности строится следующим образом. Первый шаг в сторону улучшения приверженности пациента включает оценку того, следуют ли пациенты рекомендованному им лечению. Точная оценка приверженности больных непроста, и полное понимание того, какое решение примет пациент и какова его приверженность, часто иллюзорно. Врачи, как правило, плохо информированы о приверженности их пациентов, и полагаются на собственную интуицию или на попытки «поймать» пациентов на нарушениях режима лечения, что может быть довольно проблематично. Пациенты, в свою очередь, правдиво рассказывают о своем соблюдении режима лечения только тогда, когда они не стесняются признавать наличие трудностей в вопросе приверженности лечения, что бывает только в случае отсутствия риска критики со стороны врача и в контексте реального сотрудничества с ним [21, 22]. Определение степени приверженности зависит в значительной степени от наличия доверия между пациентом и лечащим врачом, и наиболее простая система оценки, вероятно, позволяет получить наиболее честные и точные ответы [21, 42].

Реалистичная оценка знаний пациентов и понимание ими схемы лечения, их вера в нее, будет способствовать более эффективному выявлению потенциальных проблем приверженности. Многие факторы, необходимые для проведения такой оценки, являются теми элементами, которые способствуют взаимодействию и сотрудничеству при визите к врачу. Пациенты должны иметь возможность рассказать свою историю [23-27] и изложить свою точку зрения врачу. При этом врач получает много информации об убеждениях, отношении, субъективных нормах, культурном контексте, социальной поддержке, эмоциональных проблемах, связанных со здоровьем пациента (например, депрессии). Данные элементы имеют центральное значение для оценки приверженности и должны обсуждаться при общении с пациентом. Идеальное взаимопонимание не всегда достижимо, и на самом деле может не всегда быть желательным. Наличие различных точек зрения у врача и пациента может быть полезно для эффективного обсуждения вариантов лечения и способов их соблюдения $[28,29]$. Признание различий - это важная часть построения уважительных и доверительных отношений между врачами и их пациентами.

\section{ОСОБЕННОСТИ ПРИВЕРЖЕННОСТИ ЛЕЧЕНИЮ БОЛЬНЫХ САХАРНЫМ ДИАБЕТОМ 2 ТИПА}

В связи с тем, что СД2 является хроническим прогрессирующим заболеванием, для достижения терапевтических целей лечения от пациента требуется пожизненное соблюдение большого числа врачебных рекомендаций, что предопределило возникновение понятия «поведение, связанное с диабетом» [30], которое объединяет в себе не только необходимость ежедневного регулярного приема сахароснижающих лекарственных препа- ратов, но и соблюдение рекомендаций по сбалансированному питанию и физической активности, проведение регулярного самостоятельного мониторинга уровня глюкозы в крови, соблюдение правил ухода за ногами, регулярное обследование у специалистов. Отсутствие в течение длительного времени оптимального гликемического контроля приводит к развитию и прогрессированию поздних осложнений СД, что еще больше усложняет лечение больных и ухудшает качество жизни, связанное со здоровьем. Кроме того, учитывая высокую распространенность сердечно-сосудистых заболеваний, пациентам с СД2 показан пожизненный прием гиполипидемических лекарственных средств, а также, при наличии показаний, - гипотензивных и других препаратов наряду с регулярным контролем уровня артериального давления, ряда биохимических показателей. Все компоненты комплексного терапевтического подхода являются залогом успеха проводимого лечения с позиции доказательной медицины, однако лишь осознанное и мотивированное поведение пациента, а также его высокая степень информированности о важности соблюдения рекомендаций позволяют достичь желаемых результатов лечения [25].

По мнению большинства исследователей, при СД2 наблюдается неудовлетворительная (низкая) приверженность лечению, что является причиной прогрессирования осложнений (например, сердечно-сосудистых заболеваний, ретинопатии, нейропатии, нефропатии) и приводит к увеличению частоты госпитализаций и к повышению уровня смертности [28].

Низкую приверженность («неприверженность») лечению принято дифференцировать на «пассивную» и «активную» [20, 31-34].

Под «пассивной» неприверженностью лечению понимают:

- неспособность выполнения больным назначенного лечения (первичная неприверженность);

- периодическое прекращение приема лекарств (непостоянство при приеме лекарств);

- неправильный прием лекарств;

- игнорирование рекомендаций по питанию и/или регулярных лабораторных и инструментальных мониторинговых исследований;

- неправильное выполнение рекомендаций по образу жизни (например, пациент выполняет предписанные физические упражнения, но не нагружает целевую группу мышц, или он только частично избегает определенных видов продуктов, или он неправильным образом использует медицинские приборы).

Под «активной» неприверженностью лечению понимают осознанное решение пациента отклониться от назначенного курса терапии после того, как он самостоятельно оценил риски и побочные эффекты лечения в сравнении с его преимуществами.

В Кохрановском систематическом обзоре в 2005 г. был проведен анализ 21 рандомизированного клинического исследования (РКИ), посвященного проблеме повышения приверженности лечению у людей с СД2 при амбулаторном и стационарном лечении [35]. Во многих работах утверждается, что краеугольным камнем всей системы управления СД является обучение. В публикациях описываются различные структурированные программы 
обучения, но при этом до конца остается нерешенным вопрос, какой аспект обучения или какая комбинация компонентов обучения являются определяющими для улучшения приверженности. По мнению авторов обзора, необходимо понять, оказывают ли меры долгосрочный длительный эффект или обучение должно время от времени повторяться. В анализируемых работах по изучению приверженности также отсутствовал экономический анализ. В результате авторы пришли к выводу, что существующие модели по улучшению приверженности лечению у пациентов с СД2 не имеют каких-либо значительных результатов, но и не приносят вреда, и вопрос о том, способствует ли какая-нибудь стратегия повышению приверженности лечению, до сих пор остается открытым.

Haynes и соавт. также провели анализ 182 РКИ, посвященных изучению различных методов повышения приверженности лечению при СД2 [36]. По мнению авторов систематического обзора, результаты исследований противоречили друг другу, и только в некоторых из них наблюдалось одновременное улучшение приверженности и клинических результатов лечения. Предложенные в анализируемых работах методы улучшения приверженности при лечении хронических заболеваний очень сложны для реализации в рутинной клинической практике и недостаточно эффективны. Необходимы дальнейшие исследования и разработка реальных долгосрочных мероприятий по улучшению приверженности для получения статистически значимых доказательств положительного влияния приверженности на эффективность проводимой терапии.

Учитывая проблему ограниченного времени, которое может уделить врач для проведения консультации, авторы обзора предлагают, при наличии возможности, медсестрам и фармацевтам брать на себя вопросы по преодолению выявленных барьеров низкой приверженности лечению, считая это экономически эффективной стратегией. Помимо этого, значительную роль может сыграть участие в процессе лечения близкого окружения пациентов, так как социальная атмосфера крайне важна в процессе оптимизации лечения. При определении и разработке мер, способствующих повышению приверженности, эксперты рекомендуют учитывать три фактора.

1. Сложные меры, направленные на повышение приверженности, часто сравниваются с самим лечением, в то время как еще только предстоит понять, эффективнее ли они простых мер.

2. Если мероприятия показывают свою эффективность, то введение в практику всего комплекса мер должно осуществляться без требования расширения штата медицинского персонала и увеличения расходов (гибкость внедрения и внешняя валидность).

3. Если последнее не представляется возможным, то эффекты отдельных компонентов должны быть исключены, например, с помощью проведения регрессионного моделирования для того, чтобы было возможным определить наиболее прогностически значимые компоненты.

В заключение авторы систематического обзора рекомендуют всем исследователям, которые занимаются данной проблемой, перестать заново изобретать «пло- хо вращающиеся колеса мероприятий, повышающих приверженность» [36].

В нашей стране одним из ведущих специалистов в области биомедицинских и психосоциальных аспектов СД является проф. Е.Г. Старостина, которая утверждает, что «...традиционная модель оказания диабетологической помощи в своей основе имеет попытки врачей убедить пациента принять некие цели лечения, важные с медицинской точки зрения, и в соответствии с ними изменить свой образ жизни и принимать многочисленные медикаменты. Однако чаще всего в представлениях пациента, в его реальной "сиюминутной" жизни эти цели лечения не имеют такого значения, какое им придает врач. Многие больные СД не придают своему заболеванию достаточной важности, поскольку субъективная симптоматика у них нередко отсутствует, осложнения длительно протекают бессимптомно, и поэтому они испытывают особые трудности в принятии диагноза и выполнении рекомендаций, сопряженных с пожизненной терапией и изменением образа жизни. Гораздо чаще складывается обратная картина: психика пациента не выдерживает высокого уровня тревоги (по сути, созданного врачом), и после короткого периода строгого соблюдения рекомендаций происходят вытеснение неприятной информации из сознания пациента и возвращение к низкой комплаентности» [1].

В связи с этим, «...необходима новая модель взаимодействия врача и пациента, когда от больного требуется активное участие в оказании самопомощи, а врач становится для них экспертом по самоведению, помогает приобрести знания, необходимые для принятия информированных терапевтических решений, может научить методикам, необходимым для самопомощи, обеспечить социальную и эмоциональную поддержку, предложить варианты изменения поведения и стратегий совладающего поведения» [37].

К сожалению, как в нашей стране, так и в мировой практике проведение углубленного анализа индивидуальных барьеров, препятствующих выполнению рекомендаций по лечению и изменению образа жизни, может быть ограничено временем, отведенным на консультацию пациента при амбулаторном приеме. В соответствии с приказом Министерства здравоохранения РФ от 19 декабря 2016 года N 973н «Об утверждении типовых отраслевых норм времени на выполнение работ, связанных с посещением одним пациентом врача-кардиолога, врача-эндокринолога, врача-стоматолога-терапевта», на прием эндокринолога выделяется всего 19 минут [38]. За это время специалисту предлагается не только выяснить жалобы, оценить соматический статус пациента и провести скрининг на наличие поздних осложнений СД и сопутствующих заболеваний, но и проанализировать дневник больного СД, обсудить принципы диетотерапии и модификации образа жизни, подобрать соответствующую терапевтическую схему лечения, объяснив больному правила приема ССП, а также гипотензивных и гиполипидемических препаратов, выписать рецепт на льготное получение медикаментов и, наконец, занести данные пациента в электронную историю болезни и в Государственный регистр больных сахарным диабетом.

При оказании специализированной помощи больным СД2 в стационарных условиях у врача имеется возмож- 
ность разобраться не только в клинических, но и в психологических особенностях пациента, что, без сомнения, способствует формированию более высокой приверженности лечению у пациента после выписки из стационара. Однако данный вид оказания помощи является дорогостоящим и не может быть оказан всем больным с СД. Кроме того, во многих регионах имеется дефицит квалифицированных специалистов-диабетологов, а лечением больных с диабетом занимается врач общей практики.

Результатом такого подхода к лечению является сохраняющаяся высокая частота поздних осложнений СД2 в нашей стране, отсутствие компенсации углеводного обмена у 30-40\% больных на фоне низкой приверженности лечению [39].

\section{ЗАКЛЮЧЕНИЕ}

Таким образом, по мнению большинства экспертов, ни одна из существующих интервенционных стратегий (моделей поведения) не может улучшить приверженность лечению среди всех пациентов [40-42]. Успех зависит от индивидуального подхода, течения болезни и выбранного режима ведения пациента [43]. Врач должен учитывать индивидуальные психологические особенности каждого человека. Гибкость мышления, творческий подход к выбору стратегии лечения являются несомненным достоинством специалиста. Однако именно партнерские отношения врача и пациента лежат в основе достижения высокой приверженности пациента лечению. Активное участие больного в обсуждении терапевтических мероприятий, привлечение родственников, переговоры, а иногда - способность пойти на компромисс расширяют возможности для выбора варианта оптимального лечения, в ходе которого пациент берет на себя ответственность за соблюдение рекомендаций врача. Такие доверительные взаимоотношения способствуют формированию большей удовлетворенности пациентов лечению, улучшению приверженности и, в конечном счете, оказывают влияние на эффективность лечения и клинические исходы [44]. В то же время необходимо совершенствование организации специализированной медицинской помощи больным СД2, число которых с каждым годом стремительно растет и определяет бремя неинфекционной пандемии XXI в.

\section{ДОПОЛНИТЕЛЬНАЯ ИНФОРМАЦИЯ}

Конфликт интересов. Авторы декларируют отсутствие явных и потенциальных конфликтов интересов, связанных с публикацией настоящей статьи.

Участие авторов. Калашникова М.Ф. - разработка дизайна, формирование базы данных, анализ и интерпретация результатов, написание текста; Лиходей Н.В. - разработка дизайна, набор материала, формирование базы данных, анализ и интерпретация результатов, написание текста; Лиходей Е.М. - участие в разработке дизайна исследования, набор материала, анализ и интерпретация результатов, формирование базы данных, написание текста; Фадеев В.В. - редактирование, финальное утверждение рукописи.

\section{СПИСОК ЛИТЕРАТУРЫ| REFERENCES}

1. Старостина Е.Г. Роль комплаентности в ведении больных диабетом // Русский медииинский журнал. - 2015. - Т. 23. - №8. C. 477-480. [Starostina EG. Rol' komplaentnosti v vedenii bol'nykh diabetom. Russkiy meditsinskiy zhurnal. 2015;23(8):477-480. (In Russ.)]

2. Бергер М., Старостина Е.Г., Йоргенс В., Дедов И.И. Практика инсулинотерапии. - Берлин-Гейдельберг: Springer; 1995. [Berger M, Starostina EG, Jorgens V, Dedov II. Praktika insulinoterapii. Berlin-Heidelberg: Springer; 1995. (In Russ.)]

3. Sabate E.WHO Adherence Meeting Report. Geneva: World Health Organization; 2001

4. Sabate E, editor. Adherence to Long-Term Therapies: Evidence for Action. Geneva: World Health Organization; 2003.

5. Калашникова М.Ф., Бондарева И.Б., Лиходей Н.В. Приверженность лечению при сахарном диабете 2-го типа: определение понятия, современные методы оценки пациентами проводимого лечения // Лечамий врач. - 2015. - №3. - С. 27-33. [Kalashnikova MF, Bondareva IB, Likhodey NV. Commitment to the treatment in 2nd type diabetes mellitus: definition of the notion, modern methods of the treatment assessment by the patients. Practitioner. 2015;(3):27-33. (In Russ.)]

6. American College of Preventive Medicine. Medication education adherence - improving health outcomes. American College of Preventive Medicine; 2011

7. Hochbaum G, Rosenstock I, Kegels S. Health Belief Model. United States Public Health Service; 1952

8. Gellad WF, Grenard J, McGlynn EA. A Review of Barriers to Medication Adherence: A Framework for Driving Policy Options. RAND Corporation; 2009 .

9. Taha NM, Azeaz MA, Razik BGA. Factors Affecting Compliance of Diabetic Patients toward Therapeutic Management. Med J Cairo Univ. 2011;79(1):211-218

10. White JR, Jr. Improving Adherence in the Treatment of Type 2 Diabetes. US Pharm. 2010;36(4)(Compliance \& Adherence suppl.):11-15.

11. Jin J, Sklar GE, Min Sen Oh V, Chuen Li S. Factors affecting therapeutic compliance: A review from the patient's perspective. Ther Clin Risk Manag. 2008;4(1):269-286.
12. Delamater AM. Improving Patient Adherence. Clin Diabetes. 2006;24(2):71-77. doi: 10.2337/diaclin.24.2.71

13. Мошняга Е.Н. Особенности течения сахарного диабета при тревожных состояниях: Дис. канд. мед. наук. - М.; 2011. [Moshnyaga EN. Osobennosti techenija sakharnogo diabeta pri trevozhnykh sostojanijakh. [dissertation] Moscow; 2011. (In Russ.)]

14. Ajzen I, Fishbein M. Understanding attitudes and predicting social behavior. Englewood Cliffs: Prentice-Hall; 1980.

15. Strecher VJ, Champion VL, Rosenstock IM. The health belief model and health behavior. In Gochman DS, editor. Handbook of health behavior research I: Personal and social determinants. New York: Plenum Press; 1997. p. 71-91.

16. Prochaska JO, Redding CA, Evers K. The Transtheoretical Model. In: Glanz KLF, Rimer BK, editors. Health behavior and health education: theory, research, and practice. San Francisco, Jossey-Bass; 1997. p. 99-120.

17. Leventhal H, Leventhal EA, Contrada RJ. Self-regulation, health, and behavior: A perceptual-cognitive approach. Psychol Health. 1998;13(4):717-733. doi: 10.1080/08870449808407425

18. Byrne MK, Deane FP. Treatment adherence. In King R, Lloyd C, Meehan T, et al, editors. Manual of Psychosocial Rehabilitation. Oxford: Wiley-Blackwell; 2012. p. 123-134.

19. Atreja A, Bellam N, Levy SR. Strategies to enhance patient adherence: making it simple. MedGenMed. 2005;7(1):4.

20. DiMatteo MR, Haskard-Zolnierek KB, Martin LR. Improving patient adherence: a three-factor model to guide practice. Health Psychol Rev. 2012;6(1):74-91. doi: 10.1080/17437199.2010.537592

21. Haug MR, Lavin B. Practitioner or Patient - Who's in Charge? J Health Soc Behav. 1981;22(3):212. doi: 10.2307/2136517

22. Hays RD, DiMatteo MR. A short-form measure of loneliness. J Pers Assess. 1987;51(1):69-81. doi: 10.1207/s15327752jpa5101_6

23. Mishler EG. The Discourse of Medicine: Dialectics of Medical Interviews. Norwood: Ablex Publishing Corporation; 1984

24. Smith RC. The Patient's Story: Integrating the Patient- and Physician-centered Approaches to Interviewing. Ann Intern Med. 1991;115(6):470. doi: 10.7326/0003-4819-115-6-470 
25. Roter DL, Hall JA. Doctors Talking with Patients/patients Talking with Doctors: Improving Communication in Medical Visits. Westport: Auburn House; 1992.

26. Roter $\mathrm{D}$. The enduring and evolving nature of the patientphysician relationship. Patient Educ Couns. 2000;39(1):5-15. doi: 10.1016/s0738-3991(99)00086-5

27. Haidet P, Paterniti DA. "Building" a history rather than "taking" one: a perspective on information sharing during the medical interview. Arch Intern Med. 2003;163(10):1134-1140. doi: 10.1001/archinte.163.10.1134

28. Katz J. Why Doctors Don't Disclose Uncertainty. Hastings Cent Rep 1984;14(1):35. doi: 10.2307/3560848

29. Wolf SM. Conflict between Doctor and Patient. Law Med Health Care. 2016;16(3-4):197-203. doi: 10.1111/j.1748-720X.1988.tb01946.x

30. Старостина Е.Г., Галстян Г.Р., Дедов И.И. Роль поведения, связанного с диабетом, в эффективности программы лечения и обучения при сахарном диабете I типа // Проблемы эндокринологии. 1994. - T. 40. - №5. - C. 39-40. [Starostina EG, Galstjan GR, Dedov II. The role of behavior associated with diabetes in the effectiveness of the program of treatment and education of type 1 diabetes. Problems of endocrinology. 1994;40(5):39-40. (In Russ.)]

31. Antoine SL, Pieper D, Mathes T, Eikermann M. Improving the adherence of type 2 diabetes mellitus patients with pharmacy care: a systematic review of randomized controlled trials. BMC Endocr Disord. 2014;14:53. doi: 10.1186/1472-6823-14-53

32. Raum E, Kramer HU, Ruter G, et al. Medication non-adherence and poor glycaemic control in patients with type 2 diabetes mellitus. Diabetes Res Clin Pract. 2012;97(3):377-384. doi: 10.1016/j.diabres.2012.05.026

33. Rutebemberwa E, Katureebe SK, Gitta SN, et al. Perceptions of diabetes in rural areas of Eastern Uganda. Curationis. 2013;36(1):E1-7. doi: 10.4102/curationis.v36i1.121

34. Abebe SM, Berhane $Y$, Worku A. Barriers to diabetes medication adherence in North West Ethiopia. Springerplus. 2014;3:195. doi: 10.1186/2193-1801-3-195

35. Vermeire $\mathrm{E}$, Wens J, Van Royen $\mathrm{P}$, et al. Interventions for improving adherence to treatment recommendations in people with type 2 diabetes mellitus. Cochrane Database Syst Rev. 2005(2):CD003638 doi: 10.1002/14651858.CD003638.pub2

36. Haynes RB, Ackloo E, Sahota N, et al. Interventions for enhancing medication adherence. Cochrane Database Syst Rev. 2008(2):CD000011. doi: 10.1002/14651858.CD000011.pub3
37. Старостина Е.Г. Биомедицинские и психосоциальные аспекты сахарного диабета и ожирения: взаимодействие врача и пациента и пути его оптимизации: Дис. д-ра мед. наук. - М.; 2003. [Starostina EG. Biomeditsinskiye i psikhosotsial'nyye aspekty sakharnogo diabeta i ozhireniya: vzaimodeystviye vracha i patsiyenta i puti jego optimizatsii. [dissertation] Moscow; 2003. (In Russ.)]

38. Приказ Министерства здравоохранения Российской Федерации № 973н от 19 декабря 2016 г. «Об утверждении типовых отраслевых норм времени на выполнение работ, связанных с посещением одним пациентом врача-кардиолога, врача-эндокринолога, врача-стоматолога-терапевта». [Decree of the Ministry of healthcare of the Russian Federation № 973n of 19 December 2016. «Ob utverzhdenii tipovykh otraslevykh norm vremeni na vypolnenie rabot, svyazannykh s poseshcheniem odnim patsientom vracha-kardiologa, vracha-endokrinologa, vracha-stomatologa-terapevta». (In Russ.)]

39. Дедов И.И., Калашникова М.Ф., Белоусов Д.Ю., и др. Фармакоэпидемиологические аспекты мониторинга здоровья пациентов с сахарным диабетом 2 типа: результаты Российского наблюдательного многоцентрового эпидемиологического исследования ФОРСАЙТ-СД 2 // Сахарный диабет. - 2016. T. 19. - №6. - C. 443-456. [Dedov II, Kalashnikova MF, Belousov DY, et al. Assessing routine healthcare pattern for type 2 diabetes mellitus In Russia: the results of pharmacoepidemiological study (FORSIGHT-DM2). Diabetes mellitus. 2016;19(6):443-456. (In Russ.)] doi: 10.14341/DM8146

40. Hamilton GA, Toberts SJ, Johnson JM, et al. Increasing adherence in patients with primary hypertension: an intervention. Am J Health Behav. 1993;17(1):3-11.

41. Cheng TL, Ottolini MC, Baumhaft K, et al. Strategies to Increase Adherence With Tuberculosis Test Reading in a High-risk Population. Pediatrics. 1997;100(2):210-213. doi: 10.1542/peds.100.2.210

42. Roter DL, Hall JA, Merisca R, et al. Effectiveness of Interventions to Improve Patient Compliance. Med Care. 1998;36(8):1138-1161. doi: 10.1097/00005650-199808000-00004

43. MacDonald PE, Sewing S, Wang J, et al. Inhibition of Kv2.1 voltage-dependent $\mathrm{K}+$ channels in pancreatic beta-cells enhances glucose-dependent insulin secretion. J Biol Chem. 2002;277(47):44938-44945. doi: 10.1074/jbc.M205532200 I

44. Martin LR, Williams SL, Haskard KB, DiMatteo RM. The challenge of patient adherence. Ther Clin Risk Manag. 2005;1(3):189-199.

\section{ИНФОРМАЦИЯ ОБ АВТОРАХ [AUTHORS INFO]}

Калашникова Марина Федоровна, к.М.н., доцент [Marina F. Kalashnikova, MD, PhD, associate professor]; адрес: 119435, Москва, ул. Пироговская, д. 1, с. 1 [address: 1/1 Pirogovskaya street, Moscow, 119435 Russian Federation]; ORCID: http://orcid.org/0000-0002-7924-8687; eLibrary SPIN: 3777-4087; e-mail: marina_kalash@mail.ru.

Лиходей Наталья Вячеславовна [Natalia V. Likhodey]; ORCID: http://orcid.org/0000-0002-4680-0746; eLibrary SPIN: 4022-9955; e-mail: nettle_I@yahoo.com.

Лиходей Елена Максимовна [Elena M. Likhodey]; ORCID: http://orcid.org/0000-0002-8588-4938; eLibrary SPIN: 51409358; e-mail: LikhodeyEM@spa.msu.ru.

Фадеев Валентин Викторович, д.м.Н., профессор, член-корр. PAH [Valentin V. Fadeyev, MD, PhD, Professor]; ORCID: http://orcid.org/0000-0002-2504-7468; eLibrary SPIN: 6825-8417; e-mail: walfad@mail.ru.

\section{ЦИТИРОВАТЬ:}

Лиходей Н.В., Калашникова М.Ф., Лиходей Е.М., Фадеев В.В. Анализ факторов, препятствующих формированию приверженности лечению среди больных сахарным диабетом, и стратегий, способствующих ее повышению // Caхарный диабет. — 2018. — Т. 21. — №1. — C. 5-14. doi: 10.14341/DM8781

\section{TO CITE THIS ARTICLE:}

Likhodey NV, Kalashnikova MF, Likhodey EM., Fadeyev VV. Analysis of the factors that prevent adherence to treatment in patients with diabetes mellitus and the strategies that contribute to the improvement in adherence. Diabetes mellitus. 2018;21(1):5-14. doi: 10.14341/DM8781 